\title{
Novel Botrytis and Cladosporium Species Associated with Flower Diseases of Macadamia in Australia
}

\author{
Kandeeparoopan Prasannath ${ }^{1, *(\mathbb{D})}$, Roger G. Shivas ${ }^{2}$, Victor J. Galea ${ }^{3} \mathbb{D}$ and Olufemi A. Akinsanmi ${ }^{1, *(\mathbb{D})}$ \\ 1 Queensland Alliance for Agriculture \& Food Innovation, The University of Queensland, Ecosciences Precinct, \\ Dutton Park, QLD 4102, Australia \\ 2 Centre for Crop Health, University of Southern Queensland, Toowoomba, QLD 4350, Australia; \\ roger.shivas@usq.edu.au \\ 3 School of Agriculture \& Food Sciences, The University of Queensland, Gatton, QLD 4343, Australia; \\ v.galea@uq.edu.au \\ * Correspondence: k.prasannath@uq.net.au (K.P.); o.akinsanmi@uq.edu.au (O.A.A.)
}

check for updates

Citation: Prasannath, K.; Shivas, R.G.; Galea, V.J.; Akinsanmi, O.A Novel Botrytis and Cladosporium Species Associated with Flower Diseases of Macadamia in Australia. J. Fungi 2021, 7, 898. https://doi.org/ 10.3390/jof7110898

Academic Editor: Lei Cai

Received: 23 September 2021

Accepted: 21 October 2021

Published: 25 October 202

Publisher's Note: MDPI stays neutral with regard to jurisdictional claims in published maps and institutional affiliations.

Copyright: (c) 2021 by the authors. Licensee MDPI, Basel, Switzerland. This article is an open access article distributed under the terms and conditions of the Creative Commons Attribution (CC BY) license (https:// creativecommons.org/licenses/by/ $4.0 /)$.

\begin{abstract}
Macadamia (Macadamia integrifolia) is endemic to eastern Australia and produces an edible nut that is widely cultivated in commercial orchards globally. A survey of fungi associated with the grey and green mold symptoms of macadamia flowers found mostly species of Botrytis (Sclerotiniaceae, Leotiomycetes) and Cladosporium (Cladosporiaceae, Dothideomycetes). These isolates included B. cinerea, C. cladosporioides, and unidentified isolates. Amongst the unidentified isolates, one novel species of Botrytis and three novel species of Cladosporium were delimited and characterized by molecular phylogenetic analyses. The new species are Botrytis macadamiae, Cladosporium devikae, C. macadamiae, and C. proteacearum.
\end{abstract}

Keywords: Botrytis blight; Cladosporium blight; fungal ecology; raceme blight; taxonomy; tree nut

\section{Introduction}

Macadamia species and hybrids (M. integrifolia $\times$ M. tetraphylla) are native to Australia and are now grown worldwide in tropical and subtropical regions for their nuts that have edible kernels [1]. The expansion of macadamia orchards into new regions has led to an increase in the number and severity of diseases caused by fungi and oomycetes [2-4]. Flower and fruit diseases reduce the nut set and can cause significant yield losses in commercial macadamia orchards [5-7]. A mature macadamia tree can produce more than 10,000 racemes (inflorescences) during the flowering period, with 100-300 flowers per raceme $[8,9]$. Fruit and flower diseases often cause poor pollination that can reduce the nut set by $99 \%$ [10]. Diverse fungal pathogens are associated with flower blights of macadamia including Botrytis cinerea [11], Cladosporium cladosporioides [12], Neopestalotiopsis macadamiae, and Pestalotiopsis macadamiae [7].

Under high humidity and moisture, B. cinerea causes grey mold (Botrytis blight) that covers infected macadamia flowers with mycelia (Figure 1a) [11]. Index Fungorum accepted 71 Botrytis species (http:/ / www.indexfungorum.org accessed on 17 September 2021), most of which are important pathogens of a wide range of host plants, including the grapevine, tomato, strawberry, bulbous crops, and cut flowers, causing devastating diseases during the preharvest and postharvest stages [13]. Among them, B. cinerea is one of the most important plant pathogens with wide-reaching economic and scientific impacts $[14,15]$. Many new species of Botrytis have been proposed [16] since Staats et al. [17] used molecular phylogenies to recognize Botrytis spp. The genus Cladosporium (Cladosporiaceae, Dothideomycetes) was introduced by Link [18] with C. herbarum (Pers.) Link as the type species. Cladosporium cladosporioides causes flower blight known as green mold (Cladosporium blight) that manifests as olive-grey-colored mycelial patches with abundant conidia on macadamia racemes that later become necrotic (Figure 1b) [12]. Cladosporium spp. include 
endophytes, pathogens, and saprobes, and have a worldwide distribution across a range of substrates [19-23]. Cladosporium spp. are well-known as plant pathogens [19,24-26], and some can cause animal and human diseases [27-29]. Some pathogenic isolates of Cladosporium may have been wrongly classified as saprophytes, emphasizing the importance of the phylogenetic relationships for the identification of specialized lineages and cryptic species $[24,28,30]$. Some common species, C. cladosporioides, C. herbarum, and C. sphaerospermum, represent species complexes that await resolution as new isolates are collected from diverse ecosystems and geographical regions [19]. For example, C. polonicum and C. neapolitanum were described from within the $C$. cladosporioides species complex based on isolates recovered from galled flowers formed by midges on several species of Lamiaceae in Poland and Italy [31]. A phylogenetic analysis based on informative protein-coding genes is essential for the identification of species within Botrytis and Cladosporium genera [17,31].
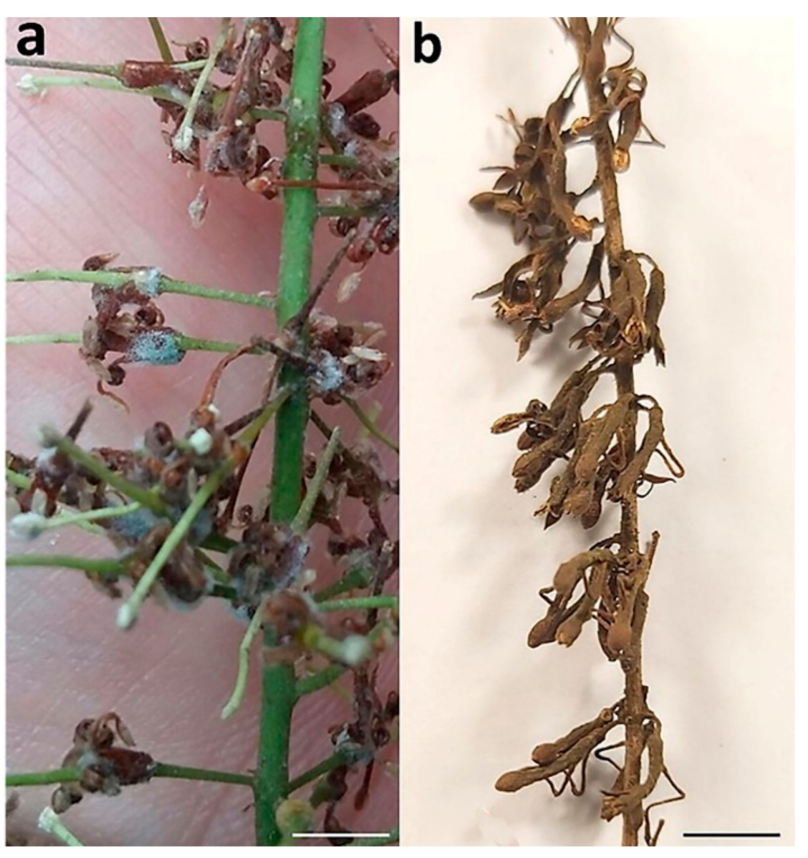

Figure 1. Macadamia racemes with symptoms of (a) grey mold, and (b) green mold. Scale bars: (a) $=5 \mathrm{~mm} ;(\mathbf{b})=10 \mathrm{~mm}$.

Macadamia is a recently domesticated tree nut crop, with only B. cinerea and C. cladosporioides in their respective genera, reported as flower blight pathogens [11,12]. However, several unidentified isolates of Botrytis and Cladosporium were obtained from macadamia racemes with grey and green mold symptoms. Therefore, this study was aimed to determine the identity of the species of Botrytis and Cladosporium that are associated with flower diseases of macadamia in Australia.

\section{Materials and Methods}

\subsection{Sample Collection and Isolation}

The isolates included in this study were obtained from macadamia racemes with symptoms of grey and green mold diseases (Table 1). Samples were collected from commercial macadamia orchards in Queensland and New South Wales, Australia in 2019 and 2020. The specimens were surface sterilized and incubated, as described by Akinsanmi et al. [7]. Monoconidial cultures of the isolates were established, as described by Akinsanmi et al. [32], and preserved in a sterile $15 \%$ glycerol solution at $-80^{\circ} \mathrm{C}$. Living cultures of the isolates were deposited in the Queensland Plant Pathology Herbarium (BRIP), Dutton Park, Australia. 
Table 1. Details of Botrytis and Cladosporium isolates obtained from macadamia racemes with flower blight symptoms included in this study.

\begin{tabular}{|c|c|c|c|c|}
\hline Isolate $^{1}$ & Species & Cultivar & Flower Growth Stage & Location $^{2}$ \\
\hline BRIP 72259a & Botrytis macadamiae & HAES 246 & 3 & Alstonville, NSW \\
\hline BRIP 72261a & B. macadamiae & HAES 246 & 3 & Alstonville, NSW \\
\hline BRIP 72276a & B. macadamiae & HAES 344 & 3 & Fernleigh, NSW \\
\hline BRIP 72295a & B. macadamiae & A16 & 3 & Knockrow, NSW \\
\hline BRIP 72278a & Cladosporium devikae & HAES 344 & 1 & Fernleigh, NSW \\
\hline BRIP 72269a & C. macadamiae & HAES 792 & 4 & Nambour, QLD \\
\hline BRIP 72287a & C. macadamiae & A16 & 3 & Maleny, QLD \\
\hline BRIP 72301a & C. proteacearum & HAES 344 & 1 & Rosebank, NSW \\
\hline
\end{tabular}

${ }^{1}$ BRIP: Queensland Plant Pathology Herbarium (BRIP) accession numbers. ${ }^{2}$ NSW: New South Wales, Australia; QLD: Queensland, Australia.

\subsection{Macro- and Micro-Morphological Studies}

Colony characteristics of cultures on a $\frac{1}{2}$-potato dextrose agar (PDA; Difco Laboratories, Franklin Lakes, NJ, USA) medium were photographed after $14 \mathrm{~d}$ of incubation at $25{ }^{\circ} \mathrm{C}$. The fungal morphology was recorded from colonies grown in the dark for $14 \mathrm{~d}$ at $25^{\circ} \mathrm{C}$ on PDA. Fungal structures were examined in lactic acid on slide mounts under a Leica DM5500B compound microscope (Wetzlar, Germany) with Nomarski differential interference contrast illumination, and images were captured with a Leica DFC 500 camera. Measurements of at least 30 conidia and other fungal structures were taken at $1000 \times$ magnification. Novel species were registered in MycoBank [33].

\subsection{DNA Extraction, PCR Amplification, and Sequencing}

Genomic DNA was extracted from approx. $40 \mathrm{mg}$ of mycelium from colonies grown on PDA for $14 \mathrm{~d}$. The mycelium was homogenized using TissueLyser (Qiagen, Chadstone, Australia) for $2 \mathrm{~min}$ at $30 \mathrm{~Hz}$, and DNA was extracted using the BioSprint 96 DNA Plant Kit on a robotic platform (Qiagen, Chadstone, Australia). The DNA concentration was determined with a BioDrop Duo spectrophotometer (BioDrop, Cambridge, UK) and adjusted to $10 \mathrm{ng} \mu \mathrm{L}^{-1}$. For Botrytis isolates, partial sequences of the glyceraldehyde 3-phosphate dehydrogenase (G3PDH) gene with primers G3PDHfor+ and G3PDHrev+ [17], DNA-dependent RNA polymerase subunit II (RPB2) gene with primers RPB2for+ and RPB2rev+ [17], and heat shock protein 60 (HSP60) gene with primers HSP60for+ and HSP60rev+ [17] were amplified. For Cladosporium isolates, amplification was carried out using primers ITS4 and ITS5 [34] for the internal transcribed spacer (ITS) region of rDNA, primers EF1-526F and EF1-1567R [35] for partial sequences of the translation elongation factor 1-alpha (TEF1 $\alpha$ ) gene, and primers ACT-512F and ACT-783R [36] for the actin (ACT) gene sequences. The DNA of each isolate served as the template for the PCR amplification. Each reaction was performed in a $25 \mu \mathrm{L}$ reaction mixture containing $5 \mu \mathrm{L}$ of $5 \times$ reaction buffer (Bioline, Eveleigh, Australia), $1.5 \mu \mathrm{L}$ of $25 \mathrm{mM} \mathrm{MgCl}_{2}, 0.5 \mu \mathrm{L}$ of $10 \mathrm{mM}$ dNTPs, $1 \mu \mathrm{L}$ each of $10 \mu \mathrm{M}$ forward and reverse primers, $0.125 \mu \mathrm{L}$ of MangoTaq DNA polymerase $(5 \mathrm{U} / \mu \mathrm{L}$; Bioline, Eveleigh, Australia), $13.875 \mu \mathrm{L}$ of nuclease free water, and $2 \mu \mathrm{L}$ of DNA template. Amplification was performed in a SuperCycler Thermal Cycler (Kyratec, Wembley, Australia) with initial denaturation at $95^{\circ} \mathrm{C}$ for $2 \mathrm{~min}$, followed by 35 cycles at $95^{\circ} \mathrm{C}$ for $30 \mathrm{~s}$, an annealing step at $55^{\circ} \mathrm{C}$ for $30 \mathrm{~s}$, and elongation at $72{ }^{\circ} \mathrm{C}$ for $1 \mathrm{~min}$, with a final extension step at $72{ }^{\circ} \mathrm{C}$ for $5 \mathrm{~min}$. The quality of PCR amplicons was checked on 1\% agarose gel electrophoresis stained with GelRed (Biotium, Melbourne, Australia) under UV light by Molecular Imager GelDoc (Bio-Rad Laboratories Inc., Gladesville, Australia). The targeted PCR products were purified and sequenced in both directions at Macrogen Inc. (Seoul, Korea).

\subsection{Phylogenetic Analyses}

The DNA sequences were assembled in Geneious Prime v. 2021.0.3 (Biomatters Ltd., San Diego, CA, USA), manually trimmed, and aligned to produce consensus sequences for each locus. The consensus sequences generated in this study were deposited in GenBank 
(Tables 2 and 3). The sequences were compared against the NCBI GenBank nucleotide database using BLASTn to determine the closest phylogenetic relatives. The sequences of the reference isolates of the Botrytis (Table 2) and Cladosporium (Table 3) species were retrieved from GenBank and aligned with the sequences generated from our isolates using MAFFT v.7.3.8.8 [37] in Geneious. Ambiguously aligned positions in each multiple alignment were excluded using Gblocks v. 0.91b [38]. The concatenated three-locus sequence dataset $(R P B 2+H S P 60+G 3 P D H)$ of Botrytis consisted of 42 taxa, with the outgroup taxon Sclerotinia sclerotiorum 484 (Table 2). The combined ITS, TEF1 $\alpha$, and ACT sequences of isolates belonging to the $C$. cladosporioides species complex comprised 72 taxa, with the outgroup taxon C. herbarum CBS 121,621 (Table 3). The combined sequence datasets were manually improved with BioEdit v. 7.2 .5 [39], and gaps were treated as missing data. Phylogenetic trees were generated from Maximum Likelihood (ML), Bayesian Inference (BI), and Maximum Parsimony (MP) analyses.

Table 2. Botrytis species and isolates used in the phylogenetic analysis with GenBank accession numbers.

\begin{tabular}{|c|c|c|c|c|}
\hline \multirow{2}{*}{ Species } & \multirow{2}{*}{ Isolate } & \multicolumn{3}{|c|}{ GenBank Accession Numbers 1} \\
\hline & & G3PDH & HSP60 & $R P B 2$ \\
\hline Botrytis aclada & MUCL8415 & AJ704992 & AJ716050 & AJ745664 \\
\hline B. allii & MUCL403 & AJ704996 & AJ716055 & AJ745666 \\
\hline B. byssoidea & MUCL94 & AJ704998 & AJ716059 & AJ745670 \\
\hline B. californica & X655 & KJ937069 & KJ937059 & KJ937049 \\
\hline B. calthae & MUCL1089 & AJ705000 & AJ716061 & AJ745672 \\
\hline B. caroliniana & CB15 & JF811584 & JF811587 & JF811590 \\
\hline B. cinerea & MUCL87 & AJ705004 & AJ716065 & AJ745676 \\
\hline B. convoluta & MUCL11595 & AJ705008 & AJ716069 & AJ745680 \\
\hline B. croci & MUCL436 & AJ705009 & AJ716070 & AJ745681 \\
\hline B. deweyae & CBS 134649 & HG799521 & HG799519 & HG799518 \\
\hline B. elliptica & BE9714 & AJ705012 & AJ716073 & AJ745684 \\
\hline B. eucalypti & CERC 7170 & KX301020 & KX301024 & KX301028 \\
\hline B. euroamericana & B83 & KC191677 & KC191678 & KC191679 \\
\hline B. fabae & MUCL98 & AJ705014 & AJ716075 & AJ745686 \\
\hline B. fabiopsis & BroadbeanBC-2 & EU519211 & EU514482 & EU514473 \\
\hline B. ficariarum & MUCL376 & AJ705016 & AJ716077 & AJ745688 \\
\hline B. fragariae & U14_P1 & KX429699 & KX429692 & KX429706 \\
\hline B. galanthina & MUCL̄435 & AJ705018 & AJ716079 & AJ745689 \\
\hline B. gladiolorum & MUCL3865 & AJ705020 & AJ716081 & AJ745692 \\
\hline B. globose & MUCL444 & AJ705022 & AJ716083 & AJ745693 \\
\hline B. hyacinthi & MUCL442 & AJ705024 & AJ716085 & AJ745696 \\
\hline \multirow[t]{4}{*}{ B. macadamiae } & BRIP 72259a & MZ344223 & MZ344234 & MZ356230 \\
\hline & BRIP 72261a & MZ344224 & MZ344235 & MZ356231 \\
\hline & BRIP 72276a & MZ344225 & MZ344236 & MZ356232 \\
\hline & BRIP 72295a & MZ344226 & MZ344237 & MZ356233 \\
\hline B. medusae & B-555 & MH732861 & MH732866 & MH732870 \\
\hline B. narcissicola & MUCL2120 & AJ705026 & AJ716087 & AJ745697 \\
\hline B. paeoniae & MUCL16084 & AJ705028 & AJ716089 & AJ745700 \\
\hline B. pelargonii & CBS497.50 & AJ704990 & AJ716046 & AM087030 \\
\hline B. polyblastis & CBS287.38 & AJ705030 & AJ716091 & AJ745702 \\
\hline B. porri & MUCL3234 & AJ705032 & AJ716093 & AJ745704 \\
\hline B. prunorum & Bpru-21 & KP339980 & KР339994 & KP339987 \\
\hline B. pseudocinerea & 10091 & JN692414 & JN692400 & JN692428 \\
\hline B. pyriformis & SedsarBC-1 & KJ543484 & KJ543488 & KJ543492 \\
\hline B. ranunculi & CBS178.63 & AJ705034 & AJ716095 & AJ745706 \\
\hline B. sinoallii & OnionBC-23 & EU519217 & EU514488 & EU514479 \\
\hline B. sinoviticola & GBC-5 & JN692413 & JN692399 & JN692427 \\
\hline B. sphaerosperma & MUCL21481 & AJ705035 & AJ716096 & AJ745708 \\
\hline B. squamosa & MUCL1107 & AJ705037 & AJ716098 & AJ745710 \\
\hline B. tulipae & BT9830 & AJ705041 & AJ716102 & AJ745713 \\
\hline Sclerotinia sclerotiorum & 484 & AJ705044 & AJ716048 & AJ745716 \\
\hline
\end{tabular}

${ }^{1}$ G3PDH: glyceraldehyde 3-phosphate dehydrogenase; HSP60: Heat shock protein 60; RPB2: DNA-dependent RNA polymerase subunit II. The name and isolates of the new species, and newly generated sequences, are shown in bold font. 
Table 3. Cladosporium species and isolates used in the phylogenetic analysis with GenBank accession numbers.

\begin{tabular}{|c|c|c|c|c|}
\hline \multirow{2}{*}{ Species } & \multirow{2}{*}{ Isolate } & \multicolumn{3}{|c|}{ GenBank Accession Numbers ${ }^{1}$} \\
\hline & & ITS & 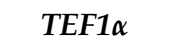 & $A C T$ \\
\hline Cladosporium acalyphae & CBS $125982^{\mathrm{T}}$ & HM147994 & HM148235 & HM148481 \\
\hline C. alboflavescens & CBS $140690^{\mathrm{T}}$ & LN834420 & LN834516 & LN834604 \\
\hline C. angulosum & CBS $140692^{\mathrm{T}}$ & LN834425 & LN834521 & LN834609 \\
\hline C. angustisporum & CBS $125983^{\mathrm{T}}$ & HM147995 & HM148236 & HM148482 \\
\hline C. angustiterminale & CBS $140480^{\mathrm{T}}$ & KT600379 & KT600476 & KT600575 \\
\hline C. anthropophilum & CBS $140685^{\mathrm{T}}$ & LN834437 & LN834533 & LN834621 \\
\hline C. arenosum & CHFC-EA 566 & MN879328 & MN890011 & MN890008 \\
\hline C. asperulatum & CBS $126340^{\mathrm{T}}$ & HM147998 & HM148239 & HM148485 \\
\hline C. australiense & CBS $125984^{\mathrm{T}}$ & HM147999 & HM148240 & HM148486 \\
\hline C. austroafricanum & CBS $140481^{\mathrm{T}}$ & KT600381 & KT600478 & KT600577 \\
\hline C. chalastosporoides & CBS $125985^{\mathrm{T}}$ & HM148001 & HM148242 & HM148488 \\
\hline C. chasmanthicola & CBS $142612^{\mathrm{T}}$ & KY646221 & KY646227 & KY646224 \\
\hline C. chubutense & CBS $124457^{\mathrm{T}}$ & FJ936158 & FJ936161 & FJ936165 \\
\hline C. cladosporioides & CBS $112388^{\mathrm{T}}$ & HM148003 & HM148244 & HM148490 \\
\hline C. colocasiae & CBS $386.64^{\mathrm{T}}$ & HM148067 & HM148310 & HM148555 \\
\hline C. colombiae & CBS $274.80 B^{\mathrm{T}}$ & FJ936159 & FJ936163 & FJ936166 \\
\hline C. crousii & CBS $140686^{\mathrm{T}}$ & LN834431 & LN834527 & LN834615 \\
\hline C. cucumerinum & CBS $171.52^{\mathrm{T}}$ & HM148072 & HM148316 & HM148561 \\
\hline C. devikae & BRIP $72278 a^{T}$ & MZ303808 & MZ344193 & MZ344212 \\
\hline C. endoviticola & JZB390018 ${ }^{\mathrm{T}}$ & MN654960 & MN984228 & MN984220 \\
\hline C. europaeum & CBS $134914^{\mathrm{T}}$ & HM148056 & HM148298 & HM148543 \\
\hline C. exasperatum & CBS $125986^{\mathrm{T}}$ & HM148090 & HM148334 & HM148579 \\
\hline C. exile & CBS $125987^{\mathrm{T}}$ & HM148091 & HM148335 & HM148580 \\
\hline C. flabelliforme & CBS $126345^{\mathrm{T}}$ & HM148092 & HM148336 & HM148581 \\
\hline C. flavovirens & CBS $140462^{\mathrm{T}}$ & LN834440 & LN834536 & LN834624 \\
\hline C. funiculosum & CBS $122129^{\mathrm{T}}$ & HM148094 & HM148338 & HM148583 \\
\hline C. gamsianum & CBS $125989^{\mathrm{T}}$ & HM148095 & HM148339 & HM148584 \\
\hline C. globisporum & CBS $812.96^{\mathrm{T}}$ & HM148096 & HM148340 & HM148585 \\
\hline C. grevilleae & CBS $114271^{\mathrm{T}}$ & JF770450 & JF770472 & JF770473 \\
\hline C. herbarum & CBS $121621^{\mathrm{T}}$ & EF679363 & EF679440 & EF679516 \\
\hline C. hillianum & CBS $125988^{\mathrm{T}}$ & HM148097 & HM148341 & HM148586 \\
\hline C. inversicolor & CBS $401.80^{\mathrm{T}}$ & HM148101 & HM148345 & HM148590 \\
\hline C. ipereniae & CBS $140483^{\mathrm{T}}$ & KT600394 & KT600491 & KT600589 \\
\hline C. iranicum & CBS $126346^{\mathrm{T}}$ & HM148110 & HM148354 & HM148599 \\
\hline C. kenpeggii & CBS $142613^{\mathrm{T}}$ & KY646222 & KY646228 & KY646225 \\
\hline C. licheniphilum & CBS $125990^{\mathrm{T}}$ & HM148111 & HM148355 & HM148600 \\
\hline C. longicatenatum & CBS $140485^{\mathrm{T}}$ & KT600403 & КT600500 & KT600598 \\
\hline \multirow[t]{2}{*}{ C. macadamiae } & BRIP $72269 \mathrm{a}^{\mathrm{T}}$ & MZ303810 & MZ344195 & MZ344214 \\
\hline & BRIP 72287a & MZ303811 & MZ344196 & MZ344215 \\
\hline C. montecillanum & CBS $140486^{\mathrm{T}}$ & KT600406 & KT600504 & KT600602 \\
\hline C. myrtacaearum & CBS $126350^{\mathrm{T}}$ & HM148117 & HM148361 & HM148606 \\
\hline C. needhamense & CBS $143359^{\mathrm{T}}$ & MF473142 & MF473570 & MF473991 \\
\hline C. neerlandicum & CBS $143360^{\mathrm{T}}$ & KP701887 & KP701764 & KP702010 \\
\hline C. neopsychrotolerans & CGMCC $3.18031^{\mathrm{T}}$ & KX938383 & KX938400 & KX938366 \\
\hline C. oxysporum & CBS 125991 & HM148118 & HM148362 & HM148607 \\
\hline C. paracladosporioides & CBS $171.54^{\mathrm{T}}$ & HM148120 & HM148364 & HM148609 \\
\hline C. parapenidielloides & CBS $140487^{\mathrm{T}}$ & KT600410 & KT600508 & KT600606 \\
\hline C. perangustum & CBS $125996^{\mathrm{T}}$ & HM148121 & HM148365 & HM148610 \\
\hline C. phaenocomae & CBS $128769^{\mathrm{T}}$ & JF499837 & JF499875 & JF499881 \\
\hline C. phyllactiniicola & CBS $126355^{\mathrm{T}}$ & HM148153 & HM148397 & HM148642 \\
\hline C. phyllophilum & CBS $125992^{\mathrm{T}}$ & HM148154 & HM148398 & HM148643 \\
\hline C. pini-ponderosae & CBS $124456^{\mathrm{T}}$ & FJ936160 & FJ936164 & FJ936167 \\
\hline C. proteacearum & BRIP 72301a ${ }^{T}$ & MZ303809 & MZ344194 & MZ344213 \\
\hline C. pseudochalastosporoides & CBS $140490^{\mathrm{T}}$ & KT600415 & KT600513 & KT600611 \\
\hline
\end{tabular}


Table 3. Cont.

\begin{tabular}{|c|c|c|c|c|}
\hline \multirow{2}{*}{ Species } & \multirow{2}{*}{ Isolate } & \multicolumn{3}{|c|}{ GenBank Accession Numbers ${ }^{1}$} \\
\hline & & ITS & TEF1 $\alpha$ & $A C T$ \\
\hline C. pseudocladosporioides & CBS $125993^{\mathrm{T}}$ & HM148158 & HM148402 & HM148647 \\
\hline C. rectoides & CBS $125994^{\mathrm{T}}$ & HM148193 & HM148438 & HM148683 \\
\hline C. rugulovarians & CBS $140495^{\mathrm{T}}$ & KT600459 & KT600558 & KT600656 \\
\hline C. scabrellum & CBS $126358^{\mathrm{T}}$ & HM148195 & HM148440 & HM148685 \\
\hline C. silenes & CBS $109082^{\mathrm{T}}$ & EF679354 & EF679429 & EF679506 \\
\hline C. sinuatum & CGMCC $3.18096^{\mathrm{T}}$ & KX938385 & KX938402 & KX938368 \\
\hline C. subuliforme & CBS $126500^{\mathrm{T}}$ & HM148196 & HM148441 & HM148686 \\
\hline C. tenuissimum & CBS $125995^{\mathrm{T}}$ & HM148197 & HM148442 & HM148687 \\
\hline C. tianshanense & CGMCC $3.18033^{\mathrm{T}}$ & KX938381 & KX938398 & KX938364 \\
\hline C. uredinicola & CPC 5390 & AY251071 & HM148467 & HM148712 \\
\hline C. uwebraunianum & CBS $143365^{\mathrm{T}}$ & MF473306 & MF473729 & MF474156 \\
\hline C. varians & CBS $126362^{\mathrm{T}}$ & HM148224 & HM148470 & HM148715 \\
\hline C. verrucocladosporioides & CBS $126363^{\mathrm{T}}$ & HM148226 & HM148472 & HM148717 \\
\hline C. vicinum & CBS $143366^{\mathrm{T}}$ & MF473311 & MF473734 & MF474161 \\
\hline C. vignae & CBS 121.25 & HM148227 & HM148473 & HM148718 \\
\hline C. welwitschiicola & CPC $18648^{\mathrm{T}}$ & KY646223 & KY646229 & KY646226 \\
\hline C. westerdijkiae & CBS $113746^{\mathrm{T}}$ & HM148061 & HM148303 & HM148548 \\
\hline C. xanthocromaticum & CBS $140691^{\mathrm{T}}$ & LN834415 & LN834511 & LN834599 \\
\hline C. xylophilum & CBS $125997^{\mathrm{T}}$ & HM148230 & HM148476 & HM148721 \\
\hline
\end{tabular}

${ }^{1}$ ITS: internal transcribed spacer; TEF1 $\alpha$ : translation elongation factor 1- $\alpha$; ACT: actin. ${ }^{\mathrm{T}}$ Ex-type isolates. The name and isolates of the new species, and newly generated sequences, are shown in bold font.

The ML analysis was implemented using RAxML v. 8.2.11 [40] in Geneious. The search option was set to rapid bootstrapping, and the analysis was run using the GTR + $\mathrm{G}+\mathrm{I}$ substitution model with 1000 bootstrap iterations. The BI analysis was conducted with MrBayes v. 3.2.1 [41] in Geneious to calculate posterior probabilities by the Markov Chain Monte Carlo (MCMC) method. The GTR + G + I evolution model was applied in the $\mathrm{BI}$ analysis. Four MCMC chains were run simultaneously, starting from random trees for $1,000,000$ generations. The temperature of the heated chain was set to 0.25 , and trees were sampled every 200 generations until the average standard deviation of split frequencies reached 0.01 (stop value). Burn-in was set at $25 \%$, after which the likelihood values were stationary. The MP analysis was performed with PAUP v. 4.0b10 [42]. Trees were inferred using a heuristic search strategy with a 100 random stepwise addition and tree-bisectionreconnection (TBR) branch swapping. Max-trees were set to 5000, and bootstrap support values were evaluated for tree branches with 1000 replications [43]. Phylograms were visualized in FigTree v. 1.4.4 [44] and annotated in Adobe Illustrator 2021.

\section{Results}

\subsection{Phylogenetic Analyses}

The concatenated sequence data matrix of Botrytis comprised 2950 base pairs (bp) (1093 for RPB2, 971 for HSP60, and 886 for G3PDH), of which 2240 bp were constant, $296 \mathrm{bp}$ were parsimony uninformative, and $414 \mathrm{bp}$ were parsimony informative. The ML analysis yielded a best scoring tree, with a final ML optimization value of $-11,930.57$ and the following model parameters: alpha-0.561, $\Pi(\mathrm{A})-0.268, \Pi(\mathrm{C})-0.241, \Pi(\mathrm{G})-0.237$, and $\Pi(\mathrm{T})-0.254$.

The combined sequence dataset of Cladosporium consisted of $1000 \mathrm{bp}$ (494 for ITS, 297 for TEF1 $\alpha$, and 209 for $A C T$ ), of which 678 bp were constant, 73 bp were parsimony uninformative, and $249 \mathrm{bp}$ were parsimony informative. The ML analysis resulted in a best scoring tree with a final ML optimization value of $-10,089.02$ and the following model parameters: alpha-0.675, $\Pi(\mathrm{A})-0.212, \Pi(\mathrm{C})-0.311, \Pi(\mathrm{G})-0.250$, and $\Pi(\mathrm{T})-0.227$.

The tree topology generated by the ML analysis was similar to that of the BI and MP analyses. The best scoring ML phylograms of Botrytis and Cladosporium are shown 
in Figures 2 and 3, respectively. ML bootstrap values, BI posterior probabilities, and MP bootstrap values (MLBS/BIPP/MPBS) are given at nodes of the phylogenetic trees (Figures 2 and 3). The phylogenetic tree inferred from the concatenated alignment resolved the four Botrytis isolates associated with the grey mold symptoms into an independent monophyletic clade with high support that represents a novel species within the Botrytis genus (Figure 2). The phylogram inferred from the combined sequence data assigned four Cladosporium isolates associated with the green mold symptoms into three well-supported monophyletic clades that represent novel species within the Cladosporium genus (Figure 3).

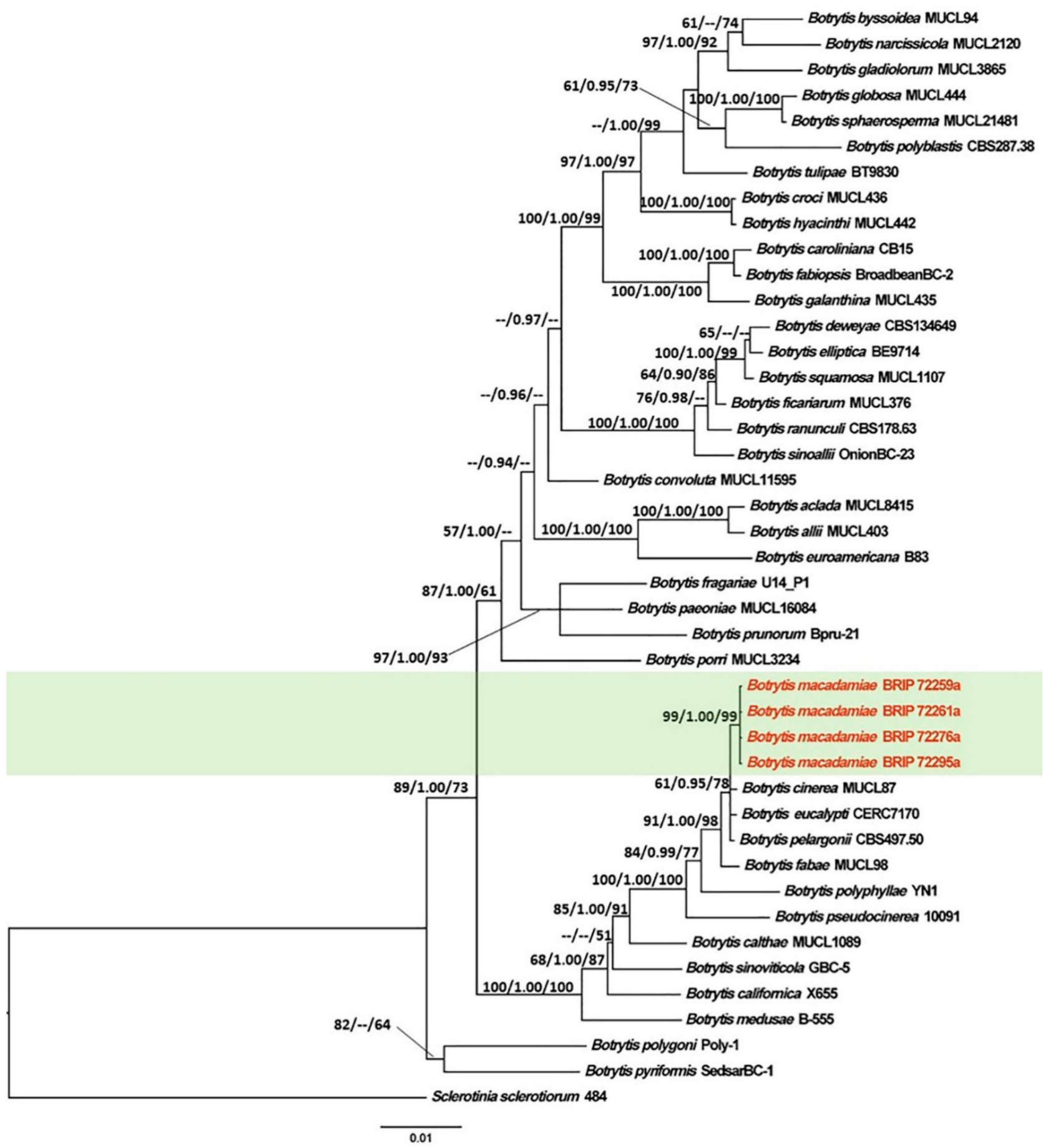

Figure 2. Maximum Likelihood tree topology of Botrytis based on a concatenated multi-locus alignment $(R P B 2+H S P 60+$ G3PDH). Sclerotinia sclerotiorum 484 was used as an outgroup taxon. Maximum Likelihood bootstrap support values ( $>50 \%)$, Bayesian Inference posterior probabilities ( $>90 \%)$, and Maximum Parsimony bootstrap proportions ( $>50 \%)$ are displayed at the nodes, respectively. Isolates of the newly described species are depicted in red. 


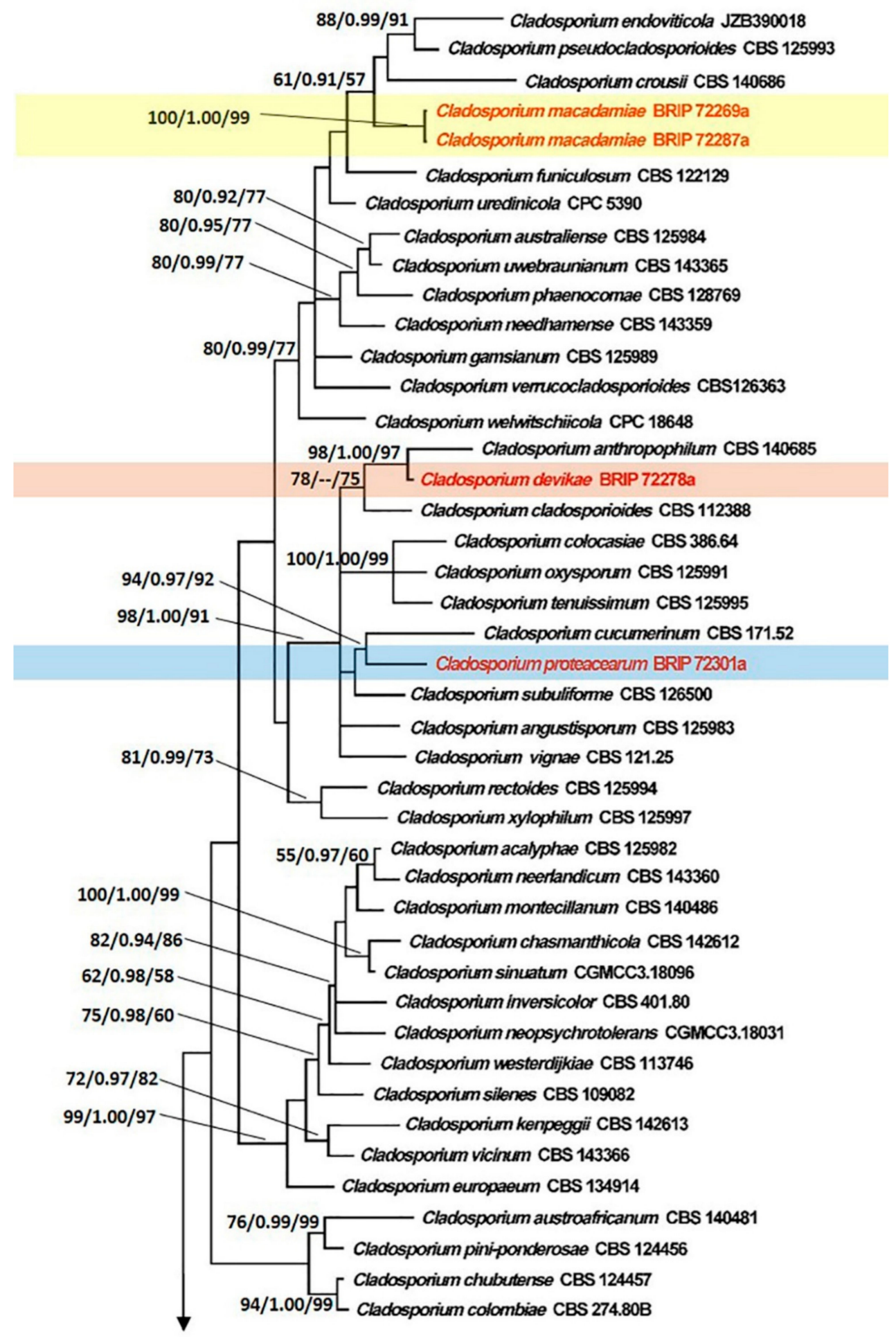

Figure 3. Cont. 


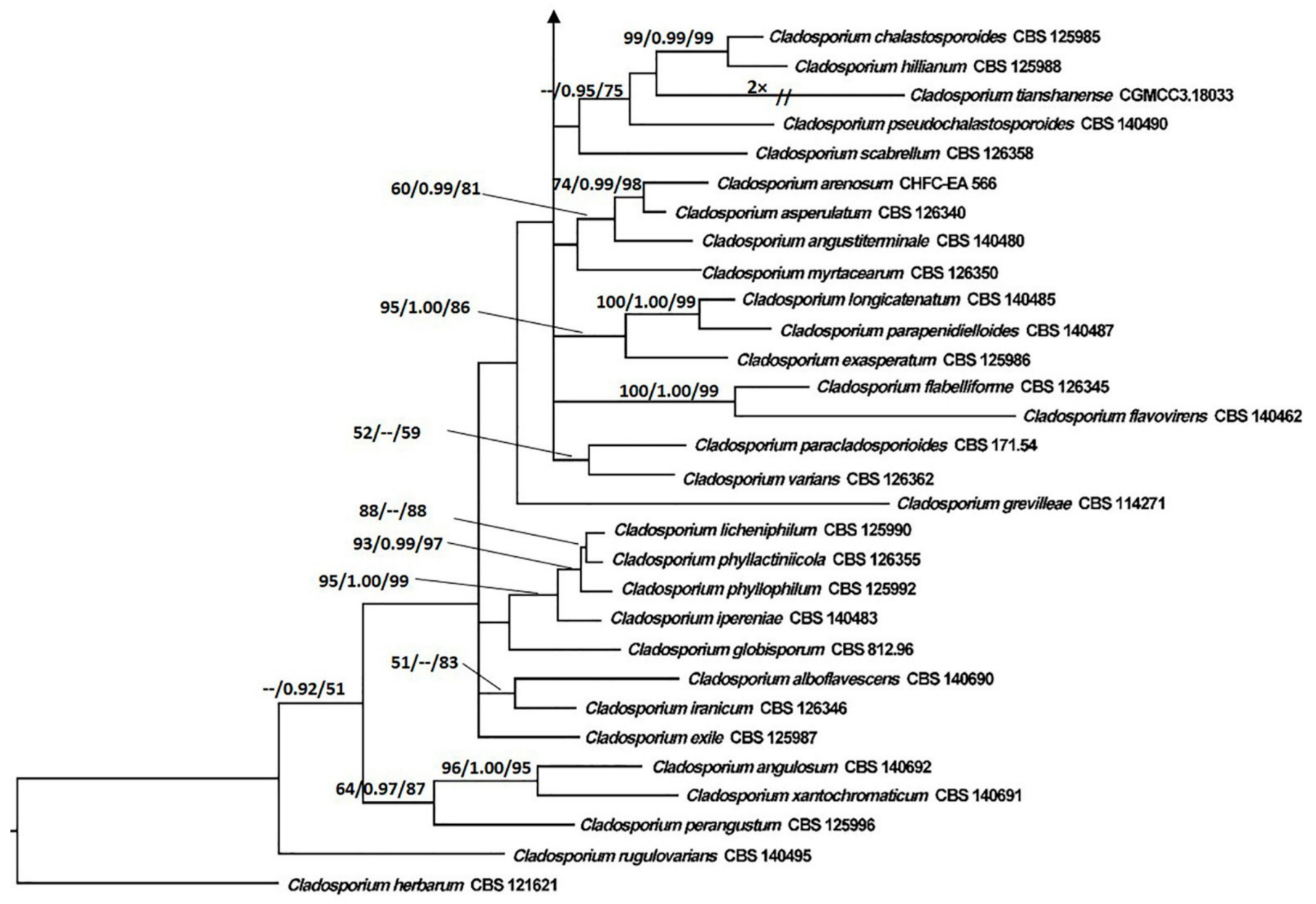

0.05

Figure 3. Maximum Likelihood tree topology of Cladosporium based on a combined multi-locus alignment $($ ITS + TEF $1 \alpha+A C T)$. Cladosporium herbarum CBS 121621 was used as an outgroup taxon. Maximum Likelihood bootstrap support values $(>50 \%)$, Bayesian Inference posterior probabilities ( $>90 \%)$, and Maximum Parsimony bootstrap proportions $(>50 \%)$ are displayed at the nodes, respectively. Isolates of the newly described species are depicted in red.

\subsection{Taxonomy}

Botrytis macadamiae Prasannath, Akinsanmi \& R.G. Shivas, sp. nov. (Figure 4). MycoBank: MB841218.

Etymology: Named after Macadamia, from which the type was first isolated.

Type: AUSTRALIA, New South Wales, Knockrow, from flower blight of Macadamia integrifolia, 25 October 2019, J. Coates (Holotype BRIP 72295a, includes ex-type culture). GenBank: MZ344226 (G3PDH); MZ344237 (HSP60); MZ356233 (RPB2).

Description: Hyphae hyaline to pale brown, septate, 3-8 $\mu \mathrm{m}$ wide. Sclerotia single, sparse, dark grey to black, irregular to spherical, immersed, scattered, $0.2-2 \mathrm{~mm}$ diam. Conidiophores branched at top, erect, septate, subhyaline to pale brown, 1020-2050 $\times 10-20 \mu \mathrm{m}$. Conidiogenous cells swollen at the apex, 10-12 × 12-14 $\mu \mathrm{m}$. Conidia in botryose clusters, elliptical to ovoid, unicellular, hyaline to pale brown, 9-11 $\times 6-7.5 \mu \mathrm{m}$.

Culture characteristics: Colonies on PDA at $25{ }^{\circ} \mathrm{C}$ after $14 \mathrm{~d}$ cover the plate, pale grey, abundant aerial mycelium in dark grey irregular tufts that cover most of the surface; reverse pale grey to buff brown.

Habitat and distribution: Racemes of Macadamia integrifolia (Proteaceae); Australia.

Other material examined: AUSTRALIA, New South Wales, Alstonville, from flower blight of Macadamia integrifolia, 17 August 2019, K. Prasannath (living cultures, BRIP 72259a 
and BRIP 72261a); AUSTRALIA, New South Wales, Fernleigh, from flower blight of Macadamia integrifolia, 23 September 2019, S. Hill (living culture, BRIP 72276a).

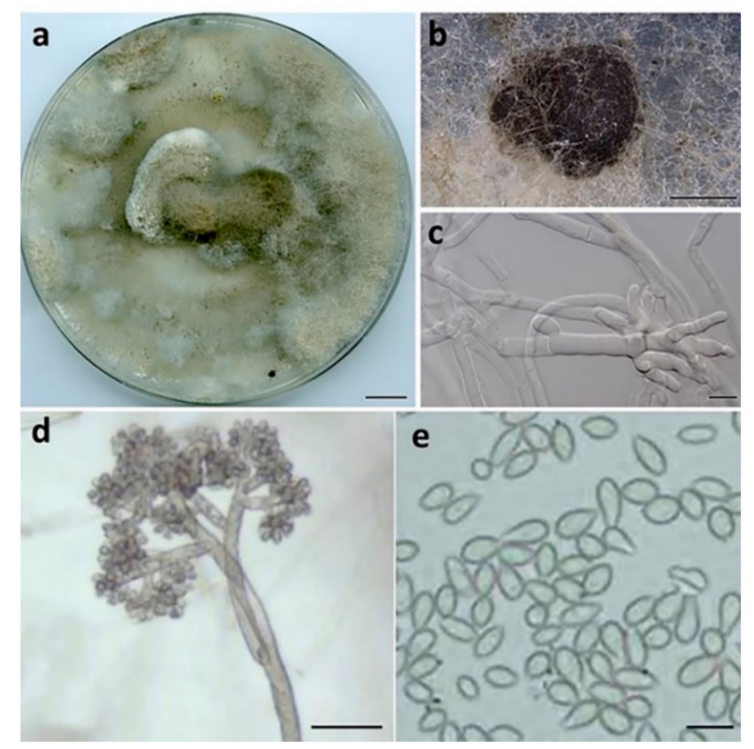

Figure 4. Botrytis macadamiae (BRIP 72295a). (a) Two-week-old colony on PDA, (b) sclerotia, (c) hyphae, (d) conidiophore, and (e) conidia. Scale bars: $(\mathbf{a})=1 \mathrm{~cm} ;(\mathbf{b})=1 \mathrm{~mm} ;(\mathbf{c}, \mathbf{e})=10 \mu \mathrm{m}$; (d) $=50 \mu \mathrm{m}$.

Notes: Botrytis macadamiae was placed in a strongly supported clade with $B$. cinerea, B. eucalypti, and B. pelargonii. BLASTn searches in GenBank showed that B. macadamiae (BRIP 72295a) differed from B. cinerea (MUCL87) in RPB2 (Identities 1070/1075, 0 gaps); from B. eucalypti (CERC 7170) in HSP60 (Identities 934/935, 0 gaps) and RPB2 (Identities 1071/1075, 0 gaps); from B. pelargonii (CBS497.50) in RPB2 (Identities 1071/1075, 0 gaps).

Cladosporium devikae Prasannath, Akinsanmi \& R.G. Shivas, sp. nov. (Figure 5).
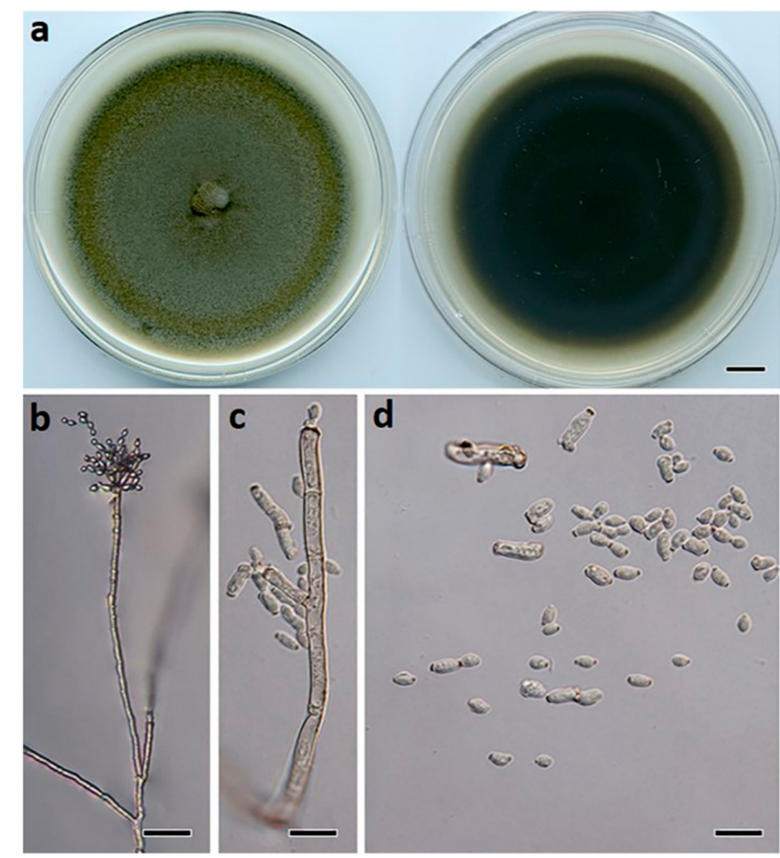

Figure 5. Cladosporium devikae (BRIP 72278a). (a) Two-week-old colony on PDA (upper surface and lower surface), (b) conidiophore, (c) ramoconidia, and (d) terminal conidia. Scale bars: $(\mathbf{a})=1 \mathrm{~cm}$; (b) $=25 \mu \mathrm{m} ;(\mathbf{c}, \mathbf{d})=10 \mu \mathrm{m}$. 
MycoBank: MB841219.

Etymology: Named after Devika Malkanthi De Costa, for her guidance and mentorship to the senior author.

Type: AUSTRALIA, New South Wales, Fernleigh, from flower blight of Macadamia integrifolia, 23 Sep. 2019, S. Hill (Holotype BRIP 72278a, includes ex-type culture). GenBank: MZ303808 (ITS); MZ344193 (TEF1 $\alpha$ ); MZ344212 (ACT).

Description: Mycelium composed of branched, septate, smooth to finely roughened, brown, 3-4.5 $\mu \mathrm{m}$ diam. hyphae. Conidiophores erect, flexuous, subcylindrical, branched and unbranched, $200-700 \times 2.5-4 \mu \mathrm{m}$, multiseptate, giving rise to an apical conidiogenous apparatus with chains of branched conidia. Primary ramoconidia subcylindrical, 11-30 $\times 3-5 \mu \mathrm{m}$, pale brown, smooth to finely roughened, 0-1-septate; hila thickened, darkened, refractive, 1.5-3.0 $\mu \mathrm{m}$ diam. Secondary ramoconidia subcylindrical to fusoid to ellipsoidal, 5-11 $\times 2-4 \mu \mathrm{m}$, pale brown, smooth to finely roughened, aseptate; hila thickened, darkened, refractive, $0.5-1.5 \mu \mathrm{m}$ diam. Intercalary and terminal conidia in branched chains (-10), ellipsoidal, 3.5-7 $\times 2-3 \mu \mathrm{m}$, subhyaline to pale brown, smooth, aseptate; hila thickened, darkened, refractive, $0.5 \mu \mathrm{m}$ diam.

Culture characteristics: Colonies on PDA $70 \mathrm{~mm}$ diam. after $14 \mathrm{~d}$ at $25^{\circ} \mathrm{C}$, flat, olivaceous, with sparse aerial mycelium, margins even and smooth; reverse black.

Habitat and distribution: Racemes of Macadamia integrifolia (Proteaceae); Australia.

Notes: Cladosporium devikae belongs to the $C$. cladosporioides species complex. Cladosporium devikae was a sister species to $C$. anthropophilum in the phylogeny. BLASTn searches in GenBank showed that $C$. devikae (BRIP 72278a) differed from C. anthropophilum ex-type (CBS 140685) in ACT (Identities 189/198, 0 gaps) and TEF1 $\alpha$ (Identities 208/217, 1 gap).

Cladosporium macadamiae Prasannath, Akinsanmi \& R.G. Shivas, sp. nov. (Figure 6).
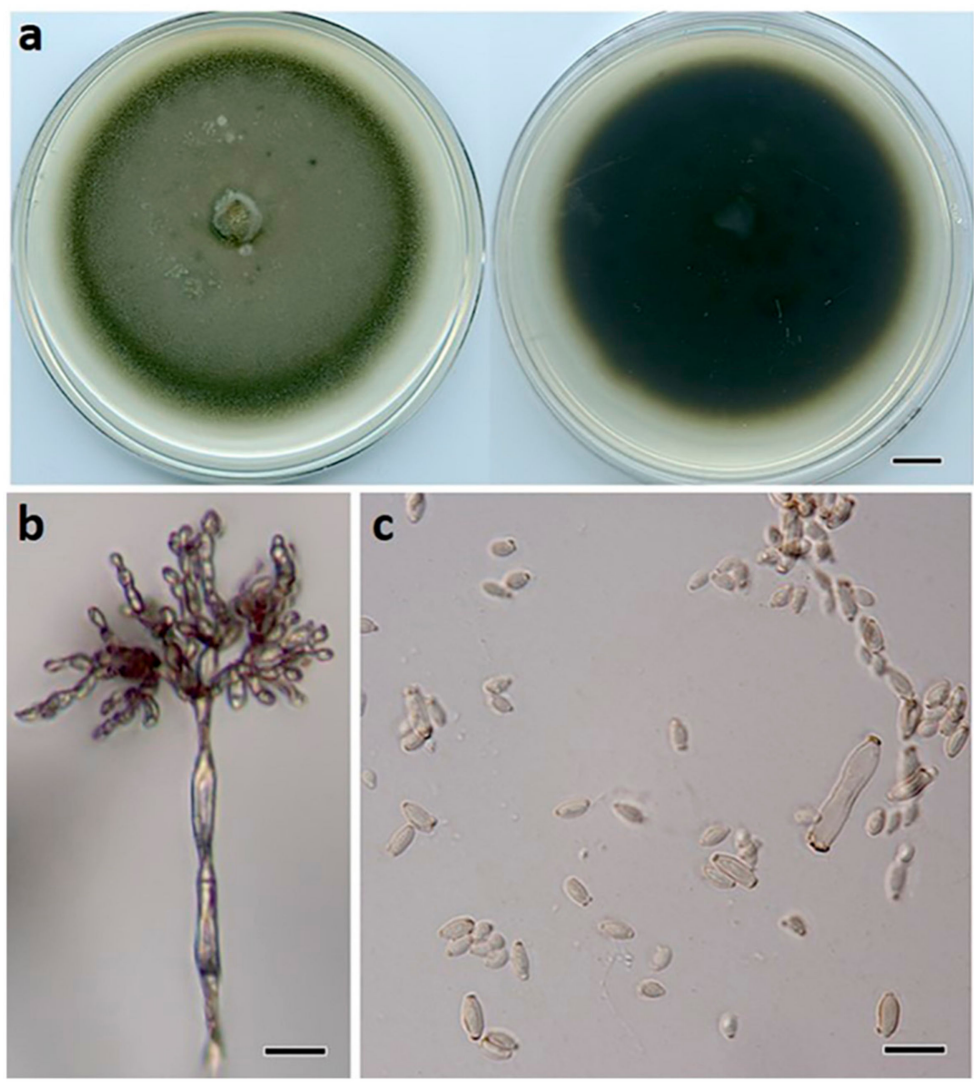

Figure 6. Cladosporium macadamiae (BRIP 72269a). (a) Two-week-old colony on PDA (upper surface and lower surface), (b) conidiophore, and (c) terminal conidia. Scale bars: $(\mathbf{a})=1 \mathrm{~cm} ;(\mathbf{b})=25 \mu \mathrm{m}$; (c) $=10 \mu \mathrm{m}$. 
MycoBank: MB841220.

Etymology: Named after Macadamia, from which the type was first isolated.

Type: AUSTRALIA, Queensland, Nambour, from flower blight of Macadamia integrifolia, 22 Aug. 2019, O.A. Akinsanmi (Holotype BRIP 72269a, includes ex-type culture). GenBank: MZ303810 (ITS); MZ344195 (TEF1 $\alpha$ ); MZ344214 (ACT).

Description: Mycelium composed of branched, septate, smooth to finely roughened, brown, 3-4.5 $\mu \mathrm{m}$ diam. hyphae. Conidiophores erect, flexuous, subcylindrical, branched and unbranched, $200-500 \times 2.5-5 \mu \mathrm{m}$, pale brown, multiseptate, giving rise to an apical conidiogenous apparatus with chains of branched conidia. Primary ramoconidia subcylindrical, 15-30 × 3-5 $\mu \mathrm{m}$, pale brown, smooth, 0-1-septate; hila thickened, darkened, refractive, 1.5-3 $\mu \mathrm{m}$ diam. Secondary ramoconidia subcylindrical to fusoid to ellipsoidal, 7-18 $\times 3-4 \mu \mathrm{m}$, pale brown, smooth, aseptate; hila thickened, darkened, refractive, $0.5-1.5 \mu \mathrm{m}$ diam. Intercalary and terminal conidia in branched chains $(-10)$, ellipsoidal, 3-7 $\times 2-3 \mu \mathrm{m}$, subhyaline to pale brown, smooth, aseptate; hila thickened, darkened, refractive, $0.5 \mu \mathrm{m}$ diam.

Culture characteristics: Colonies on PDA $70 \mathrm{~mm}$ diam. after $14 \mathrm{~d}$ at $25^{\circ} \mathrm{C}$, flat, olivaceous, with sparse aerial mycelium, margins even and smooth; reverse black.

Habitat and distribution: Racemes of Macadamia integrifolia (Proteaceae); Australia.

Other material examined: AUSTRALIA, Queensland, Maleny, from flower blight of Macadamia integrifolia, 20 September 2019, O.A. Akinsanmi (living culture, BRIP 72287a).

Notes: Cladosporium macadamiae belongs to the C. cladosporioides species complex. BLASTn searches in GenBank showed that C. macadamiae (BRIP 72269a) differed from C. crousii ex-type (CBS 140686) in ACT (Identities 199/209, 0 gaps) and TEF1 $\alpha$ (Identities 182/213, 2 gaps); from C. endoviticola ex-type (JZB390018) in ACT (Identities 153/170, 3 gaps) and TEF1 $\alpha$ (Identities 248/266, 1 gap); from C. pseudocladosporioides ex-type (CBS 125993) in ACT (Identities 197/209, 0 gaps) and TEF1 $\alpha$ (Identities 279/293, 0 gaps).

Cladosporium proteacearum Prasannath, Akinsanmi \& R.G. Shivas, sp. nov. (Figure 7).

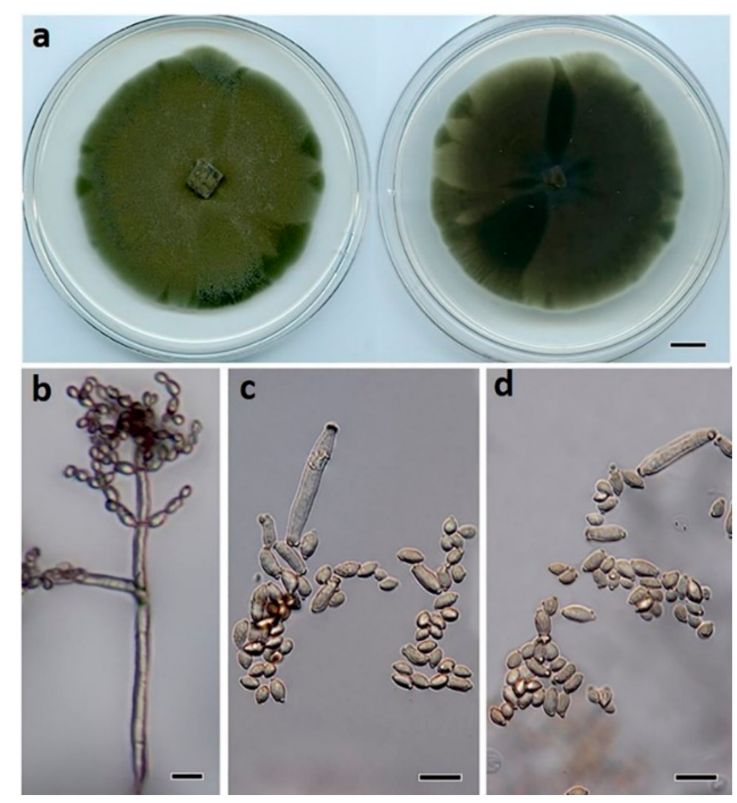

Figure 7. Cladosporium proteacearum (BRIP 72301a). (a) Two-week-old colony on PDA (upper surface and lower surface), (b) conidiophore, (c) ramoconidia, and (d) terminal conidia. Scale bars: (a) $=1 \mathrm{~cm} ;(\mathbf{b})=25 \mu \mathrm{m} ;(\mathbf{c}, \mathbf{d})=10 \mu \mathrm{m}$.

MycoBank: MB841221.

Etymology: Named after Poteaceae, the family of the host plant from which the type was first isolated. 
Type: AUSTRALIA, New South Wales, Rosebank, from flower blight of Macadamia integrifolia, 16 Oct. 2019, P. Fraser (Holotype BRIP 72301a, includes ex-type culture). GenBank: MZ303809 (ITS); MZ344194 (TEF1 $\alpha$ ); MZ344213 (ACT).

Description: Mycelium composed of branched, septate, smooth to finely roughened, brown, 3-4.5 $\mu \mathrm{m}$ diam. hyphae. Conidiophores erect, flexuous, subcylindrical, branched and unbranched, $150-500 \times 2.5-4 \mu \mathrm{m}$, multiseptate, giving rise to an apical conidiogenous apparatus with chains of branched conidia. Primary ramoconidia subcylindrical, $12-48 \times 3-5 \mu \mathrm{m}$, pale brown, smooth, 0-1-septate; hila thickened, darkened, refractive, 1.5-3 $\mu \mathrm{m}$ diam. Secondary ramoconidia subcylindrical to fusoid to ellipsoidal, 5-10 $\times 3-4 \mu \mathrm{m}$, pale brown, smooth, aseptate; hila thickened, darkened, refractive, 0.5-1.5 $\mu \mathrm{m}$ diam. Intercalary and terminal conidia in branched chains $(-10)$, ellipsoidal, $4-5 \times 2-3 \mu \mathrm{m}$, subhyaline to pale brown, smooth, aseptate; hila thickened, darkened, refractive, $0.5 \mu \mathrm{m}$ diam.

Culture characteristics: Colonies on PDA $70 \mathrm{~mm}$ diam. after $14 \mathrm{~d}$ at $25{ }^{\circ} \mathrm{C}$, flat, olivaceous, with sparse aerial mycelium, margins even and smooth; reverse olivaceous.

Habitat and distribution: Racemes of Macadamia integrifolia (Proteaceae); Australia.

Notes: Cladosporium proteacearum belongs to the C. cladosporioides species complex. Cladosporium proteacearum was a sister to C. cucumerinum in a well-supported clade. BLASTn searches in GenBank showed that C. proteacearum (BRIP 72301a) differed from C. cucumerinum ex-type (CBS 171.52) in ACT (Identities 198/209, 0 gaps); ITS (Identities 481/494, 1 gap); TEF1 $\alpha$ (Identities 274/297, 3 gaps).

\section{Discussion}

Botrytis macadamiae, Cladosporium devikae, C. macadamiae, and C. proteacearum were isolated from macadamia inflorescences with grey and green mold symptoms and subsequently described. Each species formed a well-supported monophyletic clade in the phylogenetic analysis. The ITS region of the nuclear rDNA discriminates Botrytis from other genera in Sclerotiniaceae, although ITS is not useful for the delineation of the Botrytis species [45]. The three nuclear protein-coding genes, G3PDH, HSP60, and RPB2, have been used to characterize the Botrytis species [17]. To date, 40 species are phylogenetically recognized in Botrytis $[16,46]$, including B. macadamiae. Whether B. macadamiae causes grey mold in macadamia has yet to be ascertained.

Grey mold is the most common disease caused by the Botrytis species affecting different plant organs, including flowers, fruits, leaves, and shoots [47]. Vegetables and small fruit crops such as the tomato, raspberry, grape, strawberry, blueberry, apple, and pear are among the most severely affected by these pathogens [47]. The genus Botrytis consisting of necrotrophic species has a very broad host range (e.g., B. cinerea and B. pseudocinerea) impacting more than 1400 different plant species [13]. On the contrary, other species have a narrow host range or are even host-specific, including B. fabae (broad bean) and B. calthae (marsh marigold) [48]. In some circumstances, multiple Botrytis species co-infect the same host plant; e.g., B. squamosa, B. allii, and B. aclada all cause significant economic risk to commercial onion production [15]. Interestingly, B. squamosa is family-specific and pathogenic on the onion, garlic, and leek (Allium spp.), while the closely related sister species are restricted to the lily (B. elliptica) and daylily (B. deweyae) [49]. Diversity among the Botrytis species is shown by cultural characteristics, virulence, and host specificity. However, the unique feature among all grey mold fungi is their necrotrophic lifestyle in which they kill host cells via the secretion of effector proteins to induce cell death, obtain nutrients, and subsequently colonize dead plant tissue [49,50].

The Cladosporium species are known as common and abundant fungi in indoor and outdoor environments. The Cladosporium species are also economically important spoilage organisms of grains, fruits, and refrigerated meat [51-53]. Several Cladosporium species are pathogenic to a wide range of hosts [30]. Most Cladosporium species are saprobic, but some have also been reported as endophytes, phylloplane fungi, and hyperparasites on other fungi [54-56]. Certain species show the ability to produce compounds of medical interest or are relevant as potential biocontrol agents for plant diseases $[57,58]$. Some species are 
pathogens to various crops and can cause economically important diseases, while others have only endemic importance [59]. These fungi can cause diseases of plants, often with different names, depending on the infected plants and the type of symptoms. Pathogenic species of Cladosporium are known to cause leaf mold of the tomato [60] and scab disease on leaves of the cucumber, the strawberry, and tea [61-63]. Cladosporium cladosporioides has been reported as a pathogen of scab in papaya [64], sooty mold in the persimmon [65], blossom blight in the strawberry [66], and leaf spot in the tomato [67].

Three major species complexes are recognized within the genus Cladosporium, viz. the C. cladosporioides, C. herbarum, and C. sphaerospermum species complexes [30]. The species identification and delineation in Cladosporium require a multi-locus DNA sequence analysis of the ITS region of rDNA gene, partial ACT, and TEF1 $\alpha$ gene sequences [30]. The molecular approach combined with morphological features allowed the recognition of more than 230 species within the genus Cladosporium [68]. Our phylogenetic analysis using these three loci placed C. devikae, C. macadamiae, and C. proteacearum in the C. cladosporioides species complex. These species were well-delineated from other species in the C. cladosporioides species complex.

The proper identification of species is essential for all biological studies. The present study found a high diversity of Cladosporium spp. on macadamia racemes with green mold symptoms. Future studies will determine whether B. macadamiae, C. devikae, C. macadamiae, and $C$. proteacearum are pathogens or saprobes on macadamia inflorescences. Living cultures of B. macadamiae, C. devikae, C. macadamiae, and C. proteacearum are preserved and accessible in BRIP as cryopreserved cultures for future research and study.

\section{Conclusions}

Botrytis macadamiae, Cladosporium devikae, C. macadamiae, and C. proteacearum were described and illustrated. These fungi were isolated from inflorescences of macadamia with grey and green mold symptoms in Australia. The pathogenicity of these novel species on macadamia racemes has yet to be examined. Cryopreserved isolates of these fungi are available in BRIP for future research.

Author Contributions: Methodology, data curation, molecular analysis, and writing—original draft: K.P.; taxonomy—review and editing: R.G.S.; supervision and writing-review and editing: V.J.G.; fund acquisition, lead researcher, research project administration, research concept, supervision, and writing-review and editing: O.A.A. All authors have read and agreed to the published version of the manuscript.

Funding: K.P. is a recipient of the University of Queensland Research Training Scholarship. The research was funded by Hort Innovation using the macadamia research and development levy and funds from the Australian Government-Project No. MC16018.

Institutional Review Board Statement: Not applicable.

Informed Consent Statement: Not applicable.

Data Availability Statement: All sequence data are available in NCBI GenBank (www.ncbi.nlm.nih. gov) following the accession numbers in the manuscript.

Acknowledgments: Leonardo Moryia is acknowledged for providing Figure 1a.

Conflicts of Interest: The authors declare no conflict of interest.

\section{References}

1. Hardner, C.M.; Peace, C.; Lowe, A.J.; Neal, J.; Pisanu, P.; Powell, M.; Schmidt, A.; Spain, C.; Williams, K. Genetic resources and domestication of macadamia. In Horticultural Reviews; Janick, J., Ed.; John Wiley: New York, NY, USA, 2009; pp. 1-125.

2. Jeff-Ego, O.S.; Akinsanmi, O.A. Botryosphaeriaceae causing branch dieback and tree death of macadamia in Australia. Australas. Plant Pathol. 2019, 48, 59-64. [CrossRef]

3. Jeff-Ego, O.S.; Drenth, A.; Topp, B.; Henderson, J.; Akinsanmi, O.A. Prevalence of Phytophthora species in macadamia orchards in Australia and their ability to cause stem canker. Plant Pathol. 2020, 69, 1270-1280. [CrossRef] 
4. Prasannath, K.; Galea, V.J.; Akinsanmi, O.A. Characterisation of leaf spots caused by Neopestalotiopsis clavispora and Colletotrichum siamense in macadamia in Australia. Eur. J. Plant Pathol. 2020, 156, 1219-1225. [CrossRef]

5. Akinsanmi, O.A.; Drenth, A. Economic returns from fungicide application to control husk spot of macadamia in Australia is influenced by spray efficiency, rates and costs of application. Crop Prot. 2012, 41, 35-41. [CrossRef]

6. Akinsanmi, O.A.; Drenth, A. Characterisation of husk rot in macadamia. Ann. Appl. Biol. 2017, 170, 104-115. [CrossRef]

7. Akinsanmi, O.A.; Nisa, S.; Jeff-Ego, O.S.; Shivas, R.G.; Drenth, A. Dry flower disease of macadamia in Australia caused by Neopestalotiopsis macadamiae sp. nov. and Pestalotiopsis macadamiae sp. nov. Plant Dis. 2017, 101, 45-53. [CrossRef]

8. Trueman, S.J. The reproductive biology of macadamia. Sci. Hortic. 2013, 150, 354-359. [CrossRef]

9. Trueman, S.J.; Turnbull, C.G.N. Effects of cross-pollination and flower removal on fruit set in macadamia. Ann. Bot. 1994, 73, 23-32. [CrossRef]

10. Akinsanmi, O. Flower blights of macadamia caused by Botrytis cinerea, Pestalotiopsis macadamiae and Neopestalotiopsis macadamiae in Australia. In Proceedings of the International Congress of Plant Pathology (ICPP) 2018, Boston, MA, USA, 29 July-3 August 2018.

11. Hunter, J.E.; Kunimoto, R.K. Reduction of macadamia nut set by Botrytis cinerea. Phytopathology 1973, 63, 939-941. [CrossRef]

12. Van den Berg, N.; Serfontein, S.; Christie, B.; Munro, C. First report of raceme blight caused by Cladosporium cladosporioides on macadamia nuts in South Africa. Plant Dis. 2008, 92, 484. [CrossRef]

13. Elad, Y.; Pertot, I.; Prado, A.M.C.; Stewart, A. Plant hosts of Botrytis spp. In Botrytis-The Fungus, the Pathogen and Its Management in Agricultural Systems; Fillinger, S., Elad, Y., Eds.; Springer: Cham, Switzerland, 2016; pp. 413-486.

14. Dean, R.; van Kan, J.A.L.; Pretorius, Z.A.; Hammond-Kosack, K.E.; Di Pietro, A.; Spanu, P.D.; Rudd, J.J.; Dickman, M.; Kahmann, R.; Ellis, J.; et al. The top 10 fungal pathogens in molecular plant pathology. Mol. Plant Pathol. 2012, 13, 414-430. [CrossRef] [PubMed]

15. Richards, J.K.; Xiao, C.L.; Jurick, W.M., II. Botrytis spp.: A contemporary perspective and synthesis of recent scientific developments of a widespread genus that threatens global food security. Phytopathology 2021, 111, 432-436. [CrossRef] [PubMed]

16. Garfinkel, A.R. The history of Botrytis taxonomy, the rise of phylogenetics, and implications for species recognition. Phytopathology 2021, 111, 437-454. [CrossRef]

17. Staats, M.; van Baarlen, P.; van Kan, J.A.L. Molecular phylogeny of the plant pathogenic genus Botrytis and the evolution of host specificity. Mol. Biol. Evol. 2005, 22, 333-346. [CrossRef]

18. Link, H.F. Observationes in ordines plantarum naturales. Mag. Ges. Nat. Freunde Berlin 1816, 8, $25-45$.

19. Bensch, K.; Braun, U.; Groenewald, J.Z.; Crous, P.W. The genus Cladosporium. Stud. Mycol. 2012, 72, 1-401. [CrossRef]

20. Crous, P.W.; Shivas, R.G.; Quaedvlieg, W.; van der Bank, M.; Zhang, Y.; Summerell, B.A.; Guarro, J.; Wingfield, M.J.; Wood, A.R.; Alfenas, A.C.; et al. Fungal planet description sheets: 214-280. Persoonia 2013, 31, 188. [CrossRef] [PubMed]

21. Sandoval-Denis, M.; Genã, J.; Sutton, D.A.; Wiederhold, N.P.; Cano-Lira, J.F.; Guarro, J. New species of Cladosporium associated with human and animal infections. Persoonia 2016, 36, 281-298. [CrossRef] [PubMed]

22. Tibpromma, S.; Hyde, K.D.; Mckenzie, E.H.C.; Bhat, D.J.; Phillips, A.J.L.; Wanasinghe, D.N.; Samarakoon, M.C.; Jayawardena, R.S.; Dissanayake, A.J.; Tennakoon, D.S.; et al. Fungal diversity notes 840-928: Micro-fungi associated with Pandanaceae. Fungal Divers. 2018, 72, 1-160. [CrossRef]

23. Ma, R.; Chen, Q.; Fan, Y.; Wang, Q.; Chen, S.F.; Liu, X.Z.; Cai, L.; Yao, B. Six new soil-inhabiting Cladosporium species from plateaus in China. Mycologia 2017, 109, 244-260. [CrossRef]

24. Bensch, K.; Groenewald, J.Z.; Braun, U.; Dijksterhuis, J.; de Jesús Yáñez-Morales, M.; Crous, P.W. Common but different: The expanding realm of Cladosporium. Stud. Mycol. 2015, 82, 23-74. [CrossRef] [PubMed]

25. Marin-Felix, Y.; Groenewald, J.Z.; Cai, L.; Chen, Q.; Marincowitz, S.; Barnes, I.; Bensch, K.; Braun, U.; Camporesi, E.; Damm, U.; et al. Genera of phytopathogenic fungi: GOPHY 1. Stud. Mycol. 2017, 86, 99-216. [CrossRef]

26. Rosado, A.W.C.; Custódio, F.A.; Pinho, D.B.; Ferreira, A.P.S.; Pereira, O.L. Cladosporium species associated with disease symptoms on Passiflora edulis and other crops in Brazil, with descriptions of two new species. Phytotaxa 2019, 409, 239-260. [CrossRef]

27. Velázquez-Jiménez, Y.; Hernández-Castro, R.; Romero-Romero, L.; Salas-Garrido, C.G.; Martínez-Chavarría, L.C. Feline phaeohyphomycotic cerebellitis caused by Cladosporium cladosporioides-complex: Case report and review of literature. J. Comp. Pathol. 2019, 170, 78-85. [CrossRef] [PubMed]

28. Sandoval-Denis, M.; Sutton, D.A.; Martin-Vicente, A.; Cano-Lira, J.F.; Wiederhold, N.; Guarro, J.; Gené, J. Cladosporium species recovered from clinical samples in the United States. J. Clin. Microbiol. 2015, 53, 2990-3000. [CrossRef]

29. Zhou, Y.B.; Chen, P.; Sun, T.T.; Wang, X.J.; Li, D.M. Acne-like subcutaneous phaeohyphomycosis caused by Cladosporium cladosporioides: A rare case report and review of published literatures. Mycopathologia 2016, 181, 567-573. [CrossRef] [PubMed]

30. Bensch, K.; Groenewald, J.Z.; Meijer, M.; Dijksterhuis, J.; Jurjević, Ž.; Andersen, B.; Houbraken, J.; Crous, P.W.; Samson, R.A. Cladosporium species in indoor environments. Stud. Mycol. 2018, 89, 177-301. [CrossRef]

31. Zimowska, B.; Becchimanzi, A.; Krol, E.D.; Furmanczyk, A.; Bensch, K.; Nicoletti, R. New Cladosporium species from normal and galled flowers of Lamiaceae. Pathogens 2021, 10, 369. [CrossRef]

32. Akinsanmi, O.A.; Mitter, V.; Simpfendorfer, S.; Backhouse, D.; Chakraborty, S. Identity and pathogenicity of Fusarium spp. isolated from wheat fields in Queensland and northern New South Wales. Aust. J. Agric. Res. 2004, 55, 97-107. [CrossRef]

33. Crous, P.W.; Gams, W.; Stalpers, J.A.; Robert, V.; Stegehuis, G. MycoBank: An online initiative to launch mycology into the 21st century. Stud. Mycol. 2004, 50, 19-22. 
34. White, T.J.; Bruns, T.; Lee, S.; Taylor, J. Amplification and direct sequencing of fungal ribosomal RNA genes for phylogenetics. In PCR Protocols: A Guide to Methods and Applications; Innis, M.A., Gelfand, D.H., Sninsky, J.J., White, T.J., Eds.; Academic Press: San Diego, CA, USA, 1990; pp. 315-322.

35. Rehner, S. Primers for Elongation Factor 1- $\alpha$ (EF1- $\alpha$ ); Insect Biocontrol Laboratory USDA, ARS, PSI: Beltsville, MD, USA, 2001 ; p. 4.

36. Carbone, I.; Kohn, L.M. A method for designing primer sets for speciation studies in filamentous ascomycetes. Mycologia 1999, 91, 553-556. [CrossRef]

37. Katoh, K.; Standley, D.M. MAFFT multiple sequence alignment software version 7: Improvements in performance and usability. Mol. Biol. Evol. 2013, 30, 772-780. [CrossRef] [PubMed]

38. Buffet, S.; Chevenet, F.; Dufayard, J.F.; Guindon, S.; Lefort, V.; Lescot, M.; Claverie, J.M.; Gascuel, O. Phylogeny. fr: Robust phylogenetic analysis for the non-specialist. Nucleic Acids Res. 2008, 36, W465-W469.

39. Hall, T.A. BioEdit: A user-friendly biological sequence alignment editor and analysis program for Windows 95/98/NT. Nucleic Acids Symp. Ser. 1999, 41, 95-98.

40. Stamatakis, A. RAxML version 8: A tool for phylogenetic analysis and post-analysis of large phylogenies. Bioinformatics 2014, 30, 1312-1313. [CrossRef]

41. Ronquist, F.; Huelsenbeck, J.P. MrBayes 3: Bayesian phylogenetic inference under mixed models. Bioinformatics 2003, 19, 1572-1574. [CrossRef] [PubMed]

42. Swofford, D.L. PAUP*: Phylogenetic Analysis Using Parsimony ( ${ }^{*}$ and Other Methods), Version 4.0b10; Sinauer Associates: Sunderland, MA, USA, 2002.

43. Felsenstein, J. Confidence intervals on phylogenetics: An approach using bootstrap. Evolution 1985, 39, 783-791. [CrossRef]

44. Rambaut, A. FigTree: Tree Figure Drawing Tool, Version 1.4.4; Institute of Evolutionary Biology, University of Edinburgh: Edinburgh, UK, 2018.

45. Walker, A.-S. Diversity within and between species of Botrytis. In Botrytis-The Fungus, the Pathogen and Its Management in Agricultural Systems; Fillinger, S., Elad, Y., Eds.; Springer: Cham, Switzerland, 2016; pp. 91-125.

46. He, S.Q.; Wen, Z.H.; Bai, B.; Jing, Z.Q.; Wang, X.W. Botrytis polygoni, a new species of the genus Botrytis infecting Polygonaceae in Gansu, China. Mycologia 2021, 113, 78-91. [CrossRef] [PubMed]

47. Kozhar, O.; Peever, T.L. How does Botrytis cinerea infect red raspberry? Phytopathology 2018, 108, 1287-1298. [CrossRef]

48. Plesken, C.; Weber, R.W.S.; Rupp, S.; Leroch, M.; Hahn, M. Botrytis pseudocinerea is a significant pathogen of several crop plants but susceptible to displacement by fungicide-resistant B. cinerea strains. Appl. Environ. Microbiol. 2015, 81, 7048-7056. [CrossRef] [PubMed]

49. Steentjes, M.B.F.; Scholten, O.E.; van Kan, J.A.L. Peeling the onion: Towards a better understanding of Botrytis diseases of onion. Phytopathology 2021, 111, 164-473. [CrossRef]

50. Veloso, J.; van Kan, J.A. Many shades of grey in Botrytis-host plant interactions. Trends Plant Sci. 2018, 23, 613-622. [CrossRef]

51. Fradkin, A.; Tarlo, S.M.; Tobin, R.S.; Tucic-Porretta, M.; Malloch, D. Species identification of airborne molds and its significance for the detection of indoor pollution. Air Repair 1987, 37, 51-53. [CrossRef] [PubMed]

52. Bullerman, L.B. Spoilage fungi in food-An overview. Encycl. Food Sci. Nutr. 2003, 1, 5511-5522.

53. Horner, W.E.; Worthan, A.G.; Morey, P.R. Air-and dustborne mycoflora in houses free of water damage and fungal growth. Appl. Environ. Microbiol. 2004, 70, 6394-6400. [CrossRef]

54. El-Morsy, E.M. Fungi isolated from the endorhizosphere of halophytic plants from the Red Sea Coast of Egypt. Fungal Divers. 2000, 5, 43-54.

55. Levetin, E.; Dorseys, K. Contribution to leaf surface fungi to the air spora. Aerobiologia 2006, 22, 2-12. [CrossRef]

56. Heuchert, B.; Braun, U.; Schubert, K. Morphotaxonomic revision of fungicolous Cladosporium species (Hyphomycetes). Schlechtendalia 2005, 13, 1-78.

57. Khan, M.I.H.; Sohrab, M.H.; Rony, S.R.; Tareq, F.S.; Hasan, C.M.; Mazid, M.A. Cytotoxic and antibacterial naphthoquinones from an endophytic fungus, Cladosporium sp. Toxicol. Rep. 2016, 3, 861-865. [CrossRef]

58. Adorisio, S.; Fierabracci, A.; Muscari, I.; Liberati, A.M.; Cannarile, L.; Thuy, T.T.; Sung, T.V.; Sohrab, H.; Hasan, C.M.; Ayroldi, E.; et al. Fusarubin and Anhydrofusarubin isolated from a Cladosporium species inhibit cell growth in human cancer cell lines. Toxins 2019, 11, 503. [CrossRef] [PubMed]

59. Ogórek, R.; Lejman, A.; Pusz, W.; Miłuch, A.; Miodyńska, P. Characteristics and taxonomy of Cladosporium fungi. Mikol. Lek. 2012, 19, 80-85.

60. Medina, R.; López, S.M.Y.; Franco, M.E.E.; Rollan, C.; Ronco, B.L.; Saparrat, M.C.N.; De Wit, P.J.G.M.; Balatti, P.A. A survey on occurrence of Cladosporium fulvum identifies race 0 and race 2 in tomato-growing areas of Argentina. Plant Dis. 2015, 99, 1732-1737. [CrossRef] [PubMed]

61. Lee, K.; Young, K.; Kang, H.; Ahn, K.; Min, K.; Cha, B. Cucumber scab caused by Cladosporium cucumerinum in Korea. Korean J. Plant Pathol. 1997, 13, 288-294.

62. Kwon, J.-H.; Kang, S.-W.; Kim, J.-S.; Park, C.-S. Occurrence of strawberry scab caused by Cladosporium herbarum in Korea. Mycobiology 2001, 29, 110-112. [CrossRef]

63. Kwon, J.-H.; Kang, S.-W.; Kim, J.-S.; Park, C.-S. Scab of tea (Thea sinensis) caused by Cladosporium herbarum in Korea. Plant Pathol. J. 2001, 17, 350-353. 
64. Chen, R.S.; Wang, W.L.; Li, J.C.; Wang, Y.Y.; Tsay, J.G. First report of papaya scab caused by Cladosporium cladosporioides in Taiwan. Plant Dis. 2009, 93, 426. [CrossRef]

65. Kwon, J.-H.; Park, C.-S. Sooty mold of persimmon (Diospyros kaki) caused by Cladosporium cladosporioides. Plant Pathol. J. 2003, 19, 266-268. [CrossRef]

66. Nam, M.H.; Park, M.S.; Kim, H.S.; Kim, T.I.; Kim, H.G. Cladosporium cladosporioides and C. tenuissimum cause blossom blight in strawberry in Korea. Mycobiology 2015, 43, 354-359. [CrossRef]

67. Robles-Yerena, L.; Ayala-Escobar, V.; Leyva-Mir, S.G.; Lima, N.B.; Camacho-Tapia, M.; Tovar-Pedraza, J.M. First report of Cladosporium cladosporioides causing leaf spot on tomato in Mexico. J. Plant Pathol. 2019, 101, 759. [CrossRef]

68. Iturrieta-González, I.; García, D.; Gené, J. Novel species of Cladosporium from environmental sources in Spain. MycoKeys 2021, 77, 1-25. [CrossRef] 